\title{
Isolation and TLC Densitometric Quantification of Lysergol from the Seeds of Ipomoea muricata (Linn.) Jacq.
}

\author{
Shrikant Patil, ${ }^{1}$ Manish Nivsarkar, ${ }^{2}$ and Sheetal Anandajiwala ${ }^{1}$ \\ ${ }^{1}$ Department of Natural Products, National Institute of Pharmaceutical Education and Research (NIPER) Ahmedabad, \\ B.V. Patel Pharmaceutical Education and Research Development (PERD) Centre, Sarkhej-Gandhinagar Highway, \\ Thaltej, Ahmedabad, Gujarat 380054, India \\ ${ }^{2}$ Department of Pharmacology and Toxicology, B.V. Patel Pharmaceutical Education and Research Development (PERD) Centre, \\ Sarkhej-Gandhinagar Highway, Thaltej, Ahmedabad, Gujarat 380054, India \\ Correspondence should be addressed to Sheetal Anandajiwala; drsheetalanand@gmail.com
}

Received 22 November 2012; Accepted 19 December 2012

Academic Editors: E. Boselli, S. De Marino, J. Matysiak, and A. Sanches Silva

Copyright (C) 2013 Shrikant Patil et al. This is an open access article distributed under the Creative Commons Attribution License, which permits unrestricted use, distribution, and reproduction in any medium, provided the original work is properly cited.

\begin{abstract}
Seeds of Ipomoea muricata, well known in Ayurveda for its purgative action, contains mainly indole alkaloids. Lysergol (major alkaloid) exhibits hypotensive, psychotropic, and uterus and intestine-stimulating properties. TLC fingerprint profile of $I$. muricata seeds was developed using chloroform : methanol $(95: 5 \mathrm{v} / \mathrm{v})$ as the mobile phase. Plate was visualized under UV $254 \mathrm{~nm}$ and UV $366 \mathrm{~nm}$ and after derivatization with Van Urk reagent. Lysergol resolved at $R_{f}=0.29$. Further, TLC-densitometric method was developed and validated for quantification of Lysergol avoiding derivatization step. Ethyl acetate: methanol (7:3 v/v) was used as the mobile phase. Linear regression analysis data for the calibration curve showed a good linear relationship $\left(r^{2}=0.9952\right)$ in the concentration range from $20 \mathrm{ng}$ to $140 \mathrm{ng}$, with respect to the peak area. The developed method was precise with RSD for intraday (range from 1.20 to 1.89) and interday (range from 1.39 to 1.92) for 60, 80, and $100 \mathrm{ng} / \mathrm{spot}$ of Lysergol. The instrumental precision was 0.67 (\% RSD). The limit of detection and limit of quantification for Lysergol were $12 \mathrm{ng}$ and $40 \mathrm{ng}$, respectively. The average percentage recovery was 99.68 . The amount of Lysergol was found to be $0.23 \% \mathrm{w} / \mathrm{w}$. To the best of our knowledge, this is the first report for the quantification of Lysergol from $I$. muricata seeds without derivatization.
\end{abstract}

\section{Introduction}

The seeds of Ipomoea muricata (Linn.) Jacq. (Fam. Convolvulaceae) are commonly known as Kaladana [1]. It is a well reputed drug of Ayurveda (known as Krishnabija) widely used as purgative, febrifuge, cardiac depressant, spasmolytic, hypotensive, antibacterial, and antifungal agent [1]. The seeds are used as Virechana (purgation) for its anthelmintic properties. The seeds are taken along with Saindhav (rock salt) and Shunti (Zingiber officinale); as otherwise, it gives a burning sensation (Shul) in the stomach [2]. The seeds are also incorporated in a herbal preparation known as Divya Churna (Patanjali Pharmacy, Ambala, Haryana, India) indicated for constipation, loss of appetite, and other gastric disorders [3].

Seeds of I. muricata contain $0.49 \% \mathrm{w} / \mathrm{w}$ of total alkaloids (mainly indole type) out of which Lysergol (Table 1, Scheme 1) constitutes $53 \%$ and chanoclavine $37 \%[4,5]$.
Pharmacologically the most important of the total alkaloids in I. muricata seeds are lysergic acid amide (LAA) and its epimer isolysergic acid amide (iso LAA) [5]. Lysergol has also been reported to possess hallucinogenic, hypotensive, psychotropic, analgesic, and uterus and intestine-stimulating properties [6]. It is utilized as an intermediate in the manufacture of some ergoloid medicines (e.g., nicergoline) [7] which is used to treat senile dementia and other disorders with vascular origins [8]. The seeds of I. muricata also contain resin glycosides which are laxative [1]. It also contains muricatin which gives cardiac depressant properties [9]. Recently, bioenhancing potential of Lysergol has been explored from in vitro studies, where it facilitated the transport of the antibiotics across the membrane for better efficacy on the target site [4].

There are no reports on quantification of Lysergol by TLC densitometric method. However, Genest in 1965 [5] 
TABle $1:{ }^{1} \mathrm{H}$ and ${ }^{13} \mathrm{C}$ NMR chemical shifts of Lysergol in DMSO.

\begin{tabular}{|c|c|c|c|c|}
\hline Structure of Lysergol & ${ }^{1} \mathrm{H}$ & $\delta_{\mathrm{H}}(\mathrm{ppm})$ & ${ }^{13} \mathrm{C}$ & $\delta_{\mathrm{C}}(\mathrm{ppm})$ \\
\hline & $\mathrm{H}-2$ & $6.85(\mathrm{~s}, 1 \mathrm{H})$ & $\mathrm{C}-2$ & 119 \\
\hline & $\mathrm{H}-4 \alpha$ & $2.55(\mathrm{dd}, 1 \mathrm{H})$ & $\mathrm{C}-3$ & 109.1 \\
\hline & $\mathrm{H}-4 \beta$ & $3.45(\mathrm{dd}, 1 \mathrm{H})$ & C-4 & 26.8 \\
\hline & $\mathrm{H}-5$ & $3.05(\mathrm{dd}, 1 \mathrm{H})$ & C-5 & 63.1 \\
\hline & $\mathrm{H}-7 \alpha$ & $2.2(1 \mathrm{H})$ & $\mathrm{C}-7$ & 58.8 \\
\hline & $\mathrm{H}-7 \beta$ & $3.05(\mathrm{dd}, 1 \mathrm{H})$ & $\mathrm{C}-8$ & 39.2 \\
\hline & $\mathrm{H}-8$ & $2.78(1 \mathrm{H})$ & C-9 & 122.12 \\
\hline 12 & H-9 & $6.3(\mathrm{~s}, 1 \mathrm{H})$ & C-10 & 133.7 \\
\hline & $\mathrm{H}-12$ & $7.07(2 \mathrm{H})$ & C-11 & 125.8 \\
\hline & $\mathrm{H}-13$ & $6.99(\mathrm{t}, 1 \mathrm{H})$ & C-12 & 110.8 \\
\hline & $\mathrm{H}-14$ & $7.00(2 \mathrm{H})$ & C-13 & 122.18 \\
\hline & H-18 $\alpha$ & $3.5(\mathrm{dd}, 1 \mathrm{H})$ & C-14 & 109.4 \\
\hline 15 & H-18 $\beta$ & $3.45(\mathrm{dd}, 1 \mathrm{H})$ & C-15 & 134.8 \\
\hline & $-\mathrm{N}-\mathrm{CH}_{3}$ & $2.5(\mathrm{~s}, 3 \mathrm{H})$ & C-16 & 127.8 \\
\hline $\mathrm{HN}-$ & $-\mathrm{OH}$ & $2.65(\mathrm{~s})$ & C-18 & 63.57 \\
\hline & $-\mathrm{NH}$ & $10.8(\mathrm{~s})$ & $-\mathrm{N}-\mathrm{CH}_{3}$ & 43.6 \\
\hline Lysergol & DMSO & 3.4 & & \\
\hline
\end{tabular}<smiles>[14CH3]N1CC(CO)C=C2c3cccc4[nH]cc(c34)CC21</smiles>

developed TLC fingerprint profiles of different varieties of morning glory seeds, in order to resolve a number of lysergicacid type alkaloids, including Lysergol. Lysergic acid amide, Isolysergic acid amide, and clavine alkaloids were further quantified using TLC densitometry, after derivatization with Van Urk reagent.

The present work aims to develop and validate a simple, precise, and sensitive method for the quantification of Lysergol from I. muricata seeds. Lysergol was isolated and characterized from Ipomoea muricata seeds by column chromatography. TLC fingerpint profiles were developed using Lysergol as a standard. Further, TLC densitometric method was developed and validated for the quantification of Lysergol by eliminating the derivatization step. Lysergol was quantified at a $\lambda_{\max }$ of $315 \mathrm{~nm}$ using mercury lamp in fluorescence mode.

\section{Experimental}

2.1. Plant Material. The seeds of I. muricata were procured from local market of Ahmedabad and were authenticated on the basis of its morphology and chemical test in Department of Natural products, NIPER Ahmedabad. The plant material was stored in glass bottle at $30^{\circ} \mathrm{C}$ and powdered to 40 mesh when required.

2.2. Standard Compounds. Lysergol (Purity 98\%) was received as a gift sample from Chemical Resources, Panchkula, Haryana, India.

2.3. Instrumentation. HPTLC was from Camag, Muttenz, Switzerland; UV-Vis spectrophotometer: Shimadzu-2450 (Shimadzu, Japan); LC-MS: PE SCIEX, API 165 (PerkinElmer, USA).

2.4. Isolation and Characterization of the Lysergol. Lysergol was isolated from the seed of I. muricata using column chromatography. $100 \mathrm{~g}$ of the seeds were dried, powdered, and defatted with petroleum ether for 2 hours under reflux. It was filtered, and the marc was basified with $10 \%$ ammonia solution. The marc was extracted with diethyl ether to obtain the ether fraction which was acidified using $0.1 \mathrm{~N}$ sulphuric acid. Aqueous layer was separated using separating funnel and basified with aqueous ammonia $(\mathrm{pH}=$ 9.0). The basified solution was extracted with chloroform. The chloroform fraction was collected and concentrated to get crude alkaloid $(600 \mathrm{mg})$. The crude alkaloid fraction was chromatographed over silica gel using chloroform containing increasing amounts of methanol (0-5\%). The 

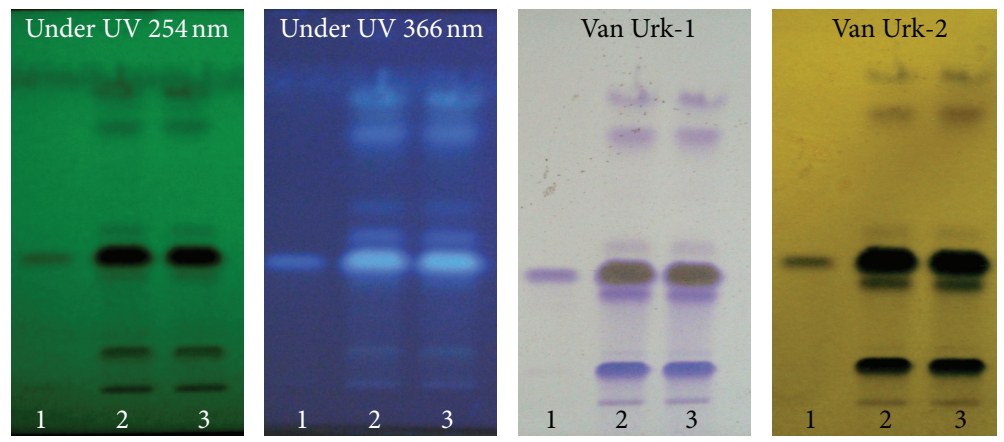

FIGURE 1: TLC fingerprint profile of Ipomoea muricata seeds. 1: Lysergol standard; 2 and 3: sample solution.

TABLE 2: Method validation parameters for the quantification of Lysergol by the proposed TLC densitometric method.

\begin{tabular}{llc}
\hline Sr. no. & Parameters & Lysergol \\
\hline 1 & Instrumental precision (\% CV, $n=7)$ & 0.67 \\
2 & Repeatability (\% CV, $n=5)$ & 1.87 \\
3 & Accuracy (average \% recovery) & 100.51 \\
4 & Limit of detection (ng) & 12 \\
5 & Limit of quantification (ng) & 40 \\
6 & Specificity & Specific \\
7 & Linearity (Correlation coefficient) & 0.9952 \\
8 & Range (ng/spot) & $40-140$ \\
9 & Linear equation & $y=99.266 x+1581.8$ \\
\hline
\end{tabular}

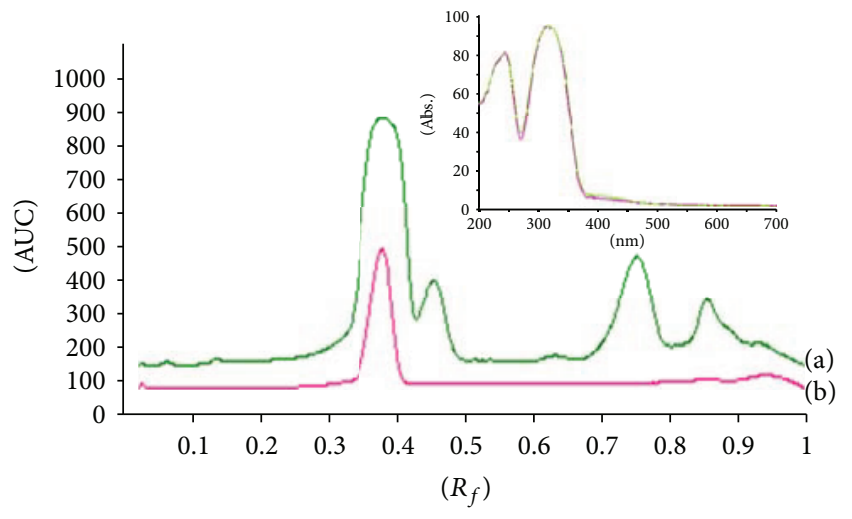

FIGURE 2: TLC densitometric chromatogram for Ipomoea muricata seeds (a) and Lysergol (b) scanned at $315 \mathrm{~nm}$. Inset: overlay of UV absorption spectra of Lysergol from sample track and standard Lysergol.

different fractions were monitored by TLC (solvent system: chloroform : methanol $(9.5: 0.5 \mathrm{v} / \mathrm{v}))$. Lysergol eluted at 5\% methanol in chloroform. All the fractions which were collected at 5\% methanol in chloroform showed a single spot at $R_{f}=0.29$. The fractions were pooled, concentrated, and evaporated to dryness under vacuum. The solid mass thus obtained was recrystallized with methanol. The isolated compound was characterized by recording the melting point (melting point apparatus, Toshniwal, India), UV absorption
TABLE 3: The intraday and interday precision for Lysergol by proposed TLC densitometric method.

\begin{tabular}{lccc}
\hline Marker & $\begin{array}{c}\text { Concentration } \\
(\mathrm{ng} / \mathrm{mL})\end{array}$ & $\begin{array}{c}\text { Intraday } \\
\text { precision* }\end{array}$ & $\begin{array}{c}\text { Interday } \\
\text { pSD })\end{array}$ \\
\hline \multirow{3}{*}{ Lysecision* } \\
& 60 & 1.20 & 1.39 \\
& 80 & 1.53 & 1.86 \\
& 100 & 1.89 & 1.92 \\
\hline
\end{tabular}

${ }^{*} \operatorname{RSD}$; mean $(n=3)$.

spectrum (UV spectrophotometer 2450, Shimadzu, Japan), IR spectrum (Shimadzu ERS 8000 FT-IR), Mass (API 165, PerkinElmer, USA), and ${ }^{1} \mathrm{H}-\mathrm{NMR}$ and ${ }^{13} \mathrm{C}$-NMR spectra (Bruker $500 \mathrm{MHz}$ NMR Spectrometer) and identified by comparing with the reported data [10]. Further confirmation was obtained by co-TLC with the standard compound.

2.5. Thin Layer Chromatography Conditions. The TLC plates were $20 \times 10 \mathrm{~cm}$, precoated with silica gel 60 F254 TLC plate (E. Merck) (0.2 mm thickness); spotting device was Camag Linomat V Automatic Sample Spotter; Camag (Muttenz, Switzerland); syringe: $100 \mu \mathrm{L}$ (Hamilton); developing chamber was a Camag glass twin trough chamber $(20 \times 10 \mathrm{~cm})$; densitometer a Camag TLC Scanner 3 linked to winCATS software. Experimental conditions: temperature was $25 \pm 2^{\circ} \mathrm{C}$ and relative humidity was $40 \%$.

2.6. Derivatization. TLC plate was derivatized with Van Urk reagent. 
TABLE 4: Recovery study of Lysergol by the proposed TLC densitometric method.

\begin{tabular}{|c|c|c|c|c|c|}
\hline Lysergol & $\begin{array}{c}\text { Amount of Lysergol } \\
\text { present (ng) }\end{array}$ & $\begin{array}{l}\text { Amount of Lysergol } \\
\text { added (ng) }\end{array}$ & $\begin{array}{l}\text { Amount Lysergol found } \\
\text { (ng) }\end{array}$ & Recovery (\%) & Average Recovery (\%) \\
\hline \multirow{3}{*}{$50 \%$ addition } & 120 & 60 & 181.37 & 100.76 & \multirow{3}{*}{$100.4 \pm 0.34$} \\
\hline & 120 & 60 & 180.91 & 100.50 & \\
\hline & 120 & 60 & 179.90 & 99.94 & \\
\hline \multirow{3}{*}{$\begin{array}{l}100 \% \\
\text { addition }\end{array}$} & 120 & 120 & 241.36 & 100.56 & \multirow{3}{*}{$100.4 \pm 0.11$} \\
\hline & 120 & 120 & 240.72 & 100.30 & \\
\hline & 120 & 120 & 240.82 & 100.34 & \\
\hline \multirow{3}{*}{$\begin{array}{l}125 \% \\
\text { addition }\end{array}$} & 120 & 150 & 271.92 & 100.71 & \multirow{3}{*}{$100.6 \pm 0.16$} \\
\hline & 120 & 150 & 272.15 & 100.79 & \\
\hline & 120 & 150 & 271.13 & 100.41 & \\
\hline
\end{tabular}

\subsection{Preparation of Van Urk Reagent [5]}

2.7.1. Van Urk-1. 2g DMBA (dimethyl amino benzaldehyde), $20 \mathrm{~mL}$ concentrated hydrochloric acid and $80 \mathrm{~mL}$ ethanol.

\subsubsection{Van Urk-2. Sodium nitrite (1\% aqueous).}

\subsection{Development of TLC Fingerprint Profiles}

2.8.1. Sample Solution. Preparation of sample solution was optimized to achieve a good fingerprinting and also to extract the marker compound efficiently. An enriched extract containing crude alkaloids was prepared as per the method reported by Genset [5]. In brief, the method can be described as follows: $2 \mathrm{~g}$ of powdered seeds were defatted by refluxing with petroleum ether $(25 \mathrm{~mL})$ for 2 hours. It was filtered, and the marc was basified with $10 \%$ ammonium hydroxide. The basified fraction was further extracted with diethyl ether, and ether layer was treated with $0.1 \mathrm{~N}$ sulphuric acid. The aqueous layer was separated using separating funnel, treated with ammonia and extracted with chloroform $(3 \times 15 \mathrm{~mL})$, and the volume was made up to $50 \mathrm{~mL}$ with chloroform in a volumetric flask.

This extract was used for TLC fingerprinting and cochromatography with marker compound.

2.8.2. Standard Solution of Lysergol. Two milligrams of Lysergol dissolved in methanol and volume were made up to $50 \mathrm{~mL}$ in volumetric flask.

2.8.3. Solvent System. Chloroform : methanol (9.5:0.5 v/v) for cochromatography with Lysergol standard.

2.8.4. Procedure. Ten microlitres of sample solution along with Lysergol standard solution were applied on a TLC plate, and the plate was developed in solvent system chloroform : methanol $(9.5: 0.5 \mathrm{v} / \mathrm{v})$ to a distance of $8 \mathrm{~cm}$. The plate was observed under UV $254 \mathrm{~nm}$ and UV $366 \mathrm{~nm}$. The purity of Lysergol band was checked by comparing absorption spectra at start, middle, and end position of isolated band of Lysergol. Identity also determined by overlaying absorption spectra of resolved band of Lysergol from sample track and standard Lysergol. The plate was then derivatized with Van Urk-1 followed by Van Urk-2 to identify and confirm indole type of alkaloids. $R_{f}$ and color of the resolved bands were noted.

\subsection{TLC Densitometric Quantification of Lysergol}

2.9.1. Sample Solution. The sample solution used for the development of fingerprint profile and cochromatography was used for quantification of Lysergol.

2.9.2. Preparation of Stock Solution of Lysergol. $20 \mu \mathrm{g} / \mathrm{mL}$ of stock solution was prepared by dissolving $1.0 \mathrm{mg}$ of accurately weighed Lysergol in methanol and made up volume of solution to $50 \mathrm{~mL}$ with methanol in volumetric flask.

2.9.3. Preparation of Standard Solution of Lysergol. The aliquots of $(2-7 \mathrm{~mL})$ stock solution were transferred to $10 \mathrm{~mL}$ volumetric flasks, and the volume of each was adjusted to $10 \mathrm{~mL}$ with methanol, to obtain standard solution containing $4,6,8,10,12$, and $14 \mu \mathrm{g} / \mathrm{mL}$ of Lysergol, respectively.

\subsubsection{Solvent System. Ethyl acetate: methanol $(7: 3 \mathrm{v} / \mathrm{v})$.}

2.9.5. Preparation of Calibration Curve of Lysergol. $10 \mu \mathrm{L}$ each of standard solution was applied in the range of 40-140 ng/spot (band width: $6 \mathrm{~mm}$, distance between the tracks: $10 \mathrm{~mm}$ ) in triplicate on a TLC plate using an automatic sample spotter. The plate was developed in a twin trough chamber $(20 \times 10 \mathrm{~cm})$ up to a distance of $8 \mathrm{~cm}$ using the mobile phase ethyl acetate: methanol $(7: 3 \mathrm{v} / \mathrm{v})$ at $25 \pm 2^{\circ} \mathrm{C}$ temperature and $40 \%$ relative humidity. The plate was dried and scanned using TLC Scanner-3 at $315 \mathrm{~nm}$ in fluorescence mode using a mercury lamp. The calibration curve of Lysergol was obtained by plotting peak areas versus applied concentrations of Lysergol.

2.9.6. Quantification of Lysergol in the Sample Solution. Ten microlitres of suitably diluted sample solution of I. muricata was applied in triplicate on a TLC plate. The plate was developed and scanned as mentioned above. The peak areas 
and absorption spectra were recorded, and the amount of Lysergol was calculated using the calibration curve.

The identity of the band of Lysergol in the sample solution was checked by overlaying their UV absorption spectra with the reference standard as Lysergol using a Camag TLC Scanner 3 with winCATS software. The purity of isolated Lysergol band in the sample track was checked by comparing the absorption spectra recorded at start, middle, and end position of the band.

2.10. Validation of the Method. ICH guidelines were followed for the validation of the analytical method developed (CPMP/ICH/281/95 and CPMP/ICH/381/95) for precision, repeatability, and accuracy.

2.10.1. Instrumental Precision. Instrumental precision was checked by repeated scanning $(n=7)$ of the same spot of Lysergol (80 ng/spot) and expressed as relative standard deviation (\% RSD).

2.10.2. Repeatability. The repeatability of the method was affirmed by analyzing $60 \mathrm{ng} / \mathrm{spot}$ of Lysergol on TLC plate $(n=5)$ and expressed as \% RSD.

2.10.3. Interday and Intraday Variation. Variability of the method was studied by analyzing aliquots of standard solution containing 60, 80, and $100 \mathrm{ng} /$ spot of Lysergol on the same day (intraday precision) and on different days (interday precision), and the results were expressed as \% RSD.

2.10.4. Limit of Detection (LOD) and Limit of Quantification (LOQ). For the evaluation of limit of detection and limit of quantification different concentrations of the standard solutions of Lysergol were applied along with methanol as blank and determined on the basis of signal to noise ratio. LOD was determined at an S/N of 3:1 and LOQ at an S/N of $10: 1$.

2.10.5. Recovery. The accuracy of the method was assessed by performing recovery study at three different levels $(50 \%$, $100 \%$, and $125 \%$ addition of Lysergol). The percent recoveries and the average percent recoveries were calculated.

2.10.6. Specificity. Specificity was ascertained by analyzing standard compounds and samples. The band for Lysergol from sample solution was confirmed by comparing the $R_{f}$ and spectra of the band to those of the standard.

\section{Results and Discussion}

There are no reports for quantification of Lysergol using HPTLC. Genset in 1965 [5] developed TLC fingerprint profiles for standard lysergic acid type alkaloids of various morning glory seeds belonging to the genus Ipomoea and Convolvulus. TLC densitometric method for quantification of Lysergic acid amide, Isolysergic acid amide, and clavine alkaloids were developed which involved the step of derivatization using Van Urk reagent. The plate after derivatization with Van Urk reagent when kept for 10-20 minutes gave more color strength than earlier seen after few minutes of derivatization, but remained steady later on, which infers that the plate has to be scanned after 20 minutes of derivatization. Moreover, it is photosensitive and has to be protected from light. Hence, derivatization with Van Urk is not only time consuming, but brings along lot of issues regarding the stability of the color developed after derivatization, which is unpredictable. This may give wrong interpretation during quantification by TLC densitometry.

During preliminary experiments, visualization of the TLC plate in the UV chamber after development suggested that Lysergol can be detected under UV $254 \mathrm{~nm}$ as well as UV $366 \mathrm{~nm}$, and the derivatization step can be avoided. Hence, in the present work, a TLC densitometric method was developed and validated for the quantification of Lysergol from $I$. muricata seeds avoiding the derivatization step.

3.1. Isolation and Characterization of Lysergol. Lysergol was isolated and characterized from seeds of $I$. muricata using column chromatography. The yield of Lysergol was found to be $167 \mathrm{mg}(1.7 \% \mathrm{w} / \mathrm{w})$. It gave bluish green colour with Van Urk reagent indicating it to be an indole type of alkaloid [5]. The melting point was recorded between $247^{\circ} \mathrm{C}$ and $249^{\circ} \mathrm{C}$ (standard Lysergol: $248^{\circ} \mathrm{C}-250^{\circ} \mathrm{C}$ ). The UV absorption maxima was found to be $315 \mathrm{~nm}$. Co-TLC with standard Lysergol using ethyl acetate: methanol $(7: 3 \mathrm{v} / \mathrm{v})$ as the solvent system gave only one band at $R_{f}$ band 0.39 . Purity of this band was confirmed by overlay of absorption spectra recorded at start, middle, and end positions of the band recorded using TLC densitometer. The IR spectrum of Lysergol exhibits the band corresponding to $\mathrm{NH}$ stretching of the pyrrole ring at $3427 \mathrm{~cm}^{-1}$. The broad signal at $3086 \mathrm{~cm}^{-1}$ corresponds to the $\mathrm{OH}$ group. The presence of tertiary amine in the structure was confirmed by the main diagnostic information gathered from the $\mathrm{C}-\mathrm{N}$ stretching vibrations $\left(1290 \mathrm{~cm}^{-1}\right)$. The characteristic $\mathrm{C}-\mathrm{H}$ stretching vibration $\left(2945 \mathrm{~cm}^{-1}\right)$ found at a lower frequency than the normal $\mathrm{C}-\mathrm{H}$ stretching frequency of methyl $\left(3320 \mathrm{~cm}^{-1}\right)$ confirms the presence of methylamino $\left(\mathrm{N}-\mathrm{CH}_{3}\right)$. The distinguishing frequencies encountered at $2873 \mathrm{~cm}^{-1}$ and $2804 \mathrm{~cm}^{-1}$ are due to two methine groups (tertiary carbon attached to hydrogen) present in the molecule. Presence of aromatic ring was confirmed by $\mathrm{C}=\mathrm{C}-\mathrm{C}$ aromatic ring stretch at $1606 \mathrm{~cm}^{-1}$ and $1446 \mathrm{~cm}^{-1}$. The mass spectrum (atmospheric pressure ionization) showed a molecular ion peak at mass-to-charge ratio $(m / z) 255.3[\mathrm{M}+\mathrm{H}]^{+}$for the molecular weight of 254.33 for Lysergol. ${ }^{1} \mathrm{H}$ NMR and ${ }^{13} \mathrm{C}$ NMR spectrum was recorded using DMSO as solvent. The chemical shift values were interpreted (Table 1). The isolated compound was confirmed as Lysergol by its spectral analysis, melting point, and coTLC.

3.2. Thin Layer Chromatography Fingerprint and Cochromatography. The TLC fingerprint profile of I. muricata seed 
extract was developed with standard Lysergol. Different sample solutions and solvent systems were tried in order to resolve maximum number of compounds. The crude alkaloid fraction was prepared and used as a sample solution. Lysergol $\left(R_{f}=0.23\right)$ along with few other compounds resolved in solvent system of chloroform: methanol $(9.5: 0.5 \mathrm{v} / \mathrm{v})$. TLC fingerprint of sample solution resolved 6 bands when observed under UV $254 \mathrm{~nm}$ and 7 bands when observed under UV $366 \mathrm{~nm}$ (Figure 1).

Ipomoea muricata seeds contain mainly indole type of alkaloids [5]. Hence, the plate was derivatized with Van Urk-1 reagent which resolved 7 compounds (purple colour). Upon further derivatization with Van Urk-2, the spots turned to dark bluish green confirming the presence of indole alkaloids [5]. Out of all the bands resolved, Lysergol showed the highest intensity, confirming it to be a major alkaloid in I. muricata seeds.

The identity of the band of Lysergol in the sample extract was confirmed by overlaying UV absorption spectra of Lysergol from sample track with that of reference standard (Lysergol) using Camag TLC Scanner 3 with winCATS software (Figure 2). The purity of Lysergol band in the sample extract track was confirmed by comparing the absorption spectra recorded at start, middle, and end positions of each of the band.

\subsection{Quantification of Lysergol HPTLC (Using High Perfor-} mance Thin Layer Chromatography). The marker compound as Lysergol was quantified from I. muricata by TLC densitometric method using HPTLC. The developed method was validated as per ICH guidelines (CPMP/ICH/281/95 and $\mathrm{CPMP} / \mathrm{ICH} / 381 / 95)$ in terms of precision, repeatability, and accuracy (Tables 2-4). The linearity was found in the range of $40-140 \mathrm{ng} / \mathrm{spot}$ (Table 1) with correlation coefficient $(r$ value) of 0.995 . The densitometric method was found to be precise with RSD for intraday from 1.20 to 1.89 , and for interday, it was from 1.39 to 1.92 for 60,80 , and $100 \mathrm{ng} / \mathrm{spot}$ of Lysergol (Table 3). This indicates that the proposed method was precise and reproducible. The LOD and LOQ for Lysergol were 12 and $40 \mathrm{ng}$, respectively. The average recovery was found to be $100.51 \%$ (Tables 2 and 4 ).

The amount of Lysergol was found to be $0.23 \% \mathrm{w} / \mathrm{w}$ in the sample analyzed (Figure 2).

\section{Conclusion}

A simple method was developed for isolation of Lysergol from Ipomoea muricata seeds using column chromatography. Further, a TLC densitometric method was developed and validated for the quantification of Lysergol from Ipomoea muricata seeds, avoiding derivatization step. The developed method for the quantification of Lysergol was found to be simple, precise, accurate, and time saving. The developed method can be used for the quantification of Lysergol from herbal raw materials and formulations containing I. muricata and can also aid in maintaining the quality of I. muricata seeds.

\section{Acknowledgments}

The authors are thankful to NIPER-Ahmedabad and B. V. Patel Pharmaceutical Education and Research Development (PERD) Centre, Ahmedabad, Gujarat, India for providing the facility for successful completion of the work. The authors wish to acknowledge Mr. Pavan Gohel, Chemical Resources, Panchkula, Haryana, India, for a gift sample of standard Lysergol and Mr. Baljinder Singh, IIIM Jammu for his help in NMR studies.

\section{References}

[1] C. P. Khare, Indian Medicinal Plant: An Illustrated Dictionary, Springer, Berlin, Germany, 2007.

[2] B. Vaidya, Nighantu Adarsh, Choukhambha Bharati Academy, Varanasi, India, 1999.

[3] http://www.swamiramdevmedicines.com/.

[4] S. P. S. Khanuja, S. Shrivastav, A. Shasani, S. Kumar, and S. Kumar, "Antibiotic pharmaceutical composition with Lysergol as bionenhancer and method of treatment," United States Patent Application Publication, Pub.no. US2007/0060604A1, March 2007.

[5] K. Genest, "A direct densitometric method on thin-layer plates for the determination of lysergic acid amide, isolysergic acid amide and clavine alkaloids in morning glory seeds," Journal of Chromatography, vol. 19, pp. 531-539, 1965.

[6] G. Ferrari, "Method for the extraction of lysergol and ergot alkaloids from plants of the Ipomoea genus," US Patent 3, 920, 663, 1975.

[7] D. Grocer and G. F. Heinz, "Biochemistry of ergot alkaloids-achievements and challenges," in The Alkaloids: Chemistry and Biology, G. A. Cordell, Ed., vol. 50, pp. 171-218, Academic Press, San Diego, Calif, USA, 1998.

[8] B. Saletu, E. Paulus, L. Linzmayer et al., "Nicergoline in senile dementia of Alzheimer type and multi-infarct dementia: a double blind, placebo controlled, clinical and EEG/ERP mapping study," Psychopharmacology, vol. 117, no. 4, pp. 385-395, 1995.

[9] M. L. Gupta, J. P. Tewari, S. N. Khanna, P. C. Gupta, M. C. Srivastava, and S. S. Mishra, "Phytopharmacologic studies of Ipomoea muricata seeds," Journal of Pharmaceutical Sciences, vol. 56, no. 6, pp. 771-773, 1967.

[10] V. Křen, P. Olšovský, V. Havlíček, P. Sedmera, M. Witvrouw, and E. de Clercq, " $N$-deoxyribosides of ergot alkaloids: synthesis and biological activity," Tetrahedron, vol. 53, no. 12, pp. 4503-4510, 1997. 

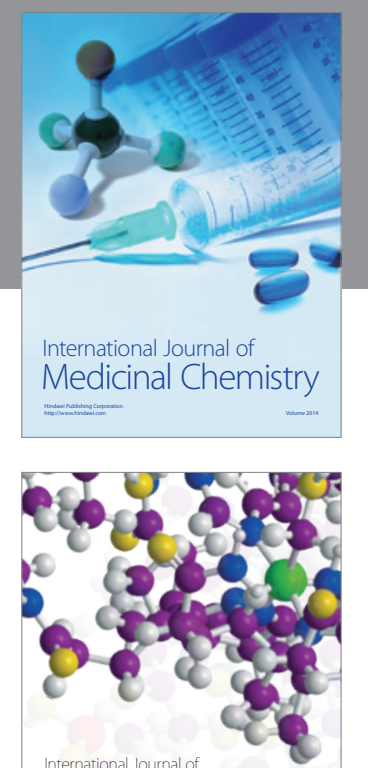

\section{Carbohydrate} Chemistry

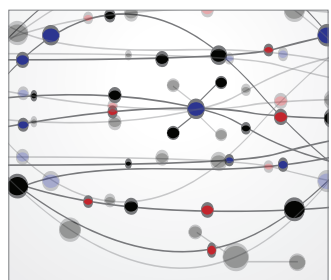

The Scientific World Journal
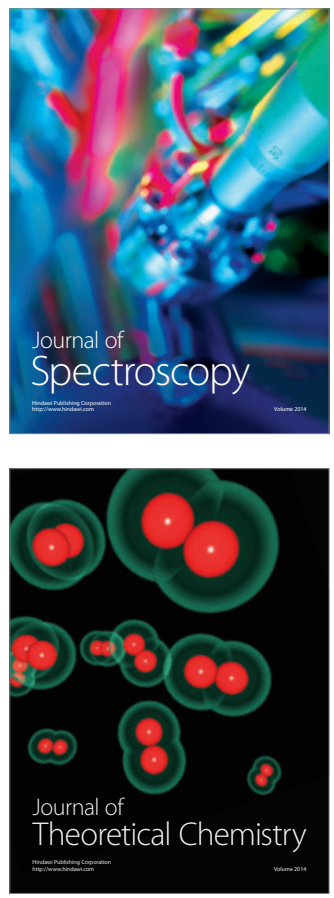
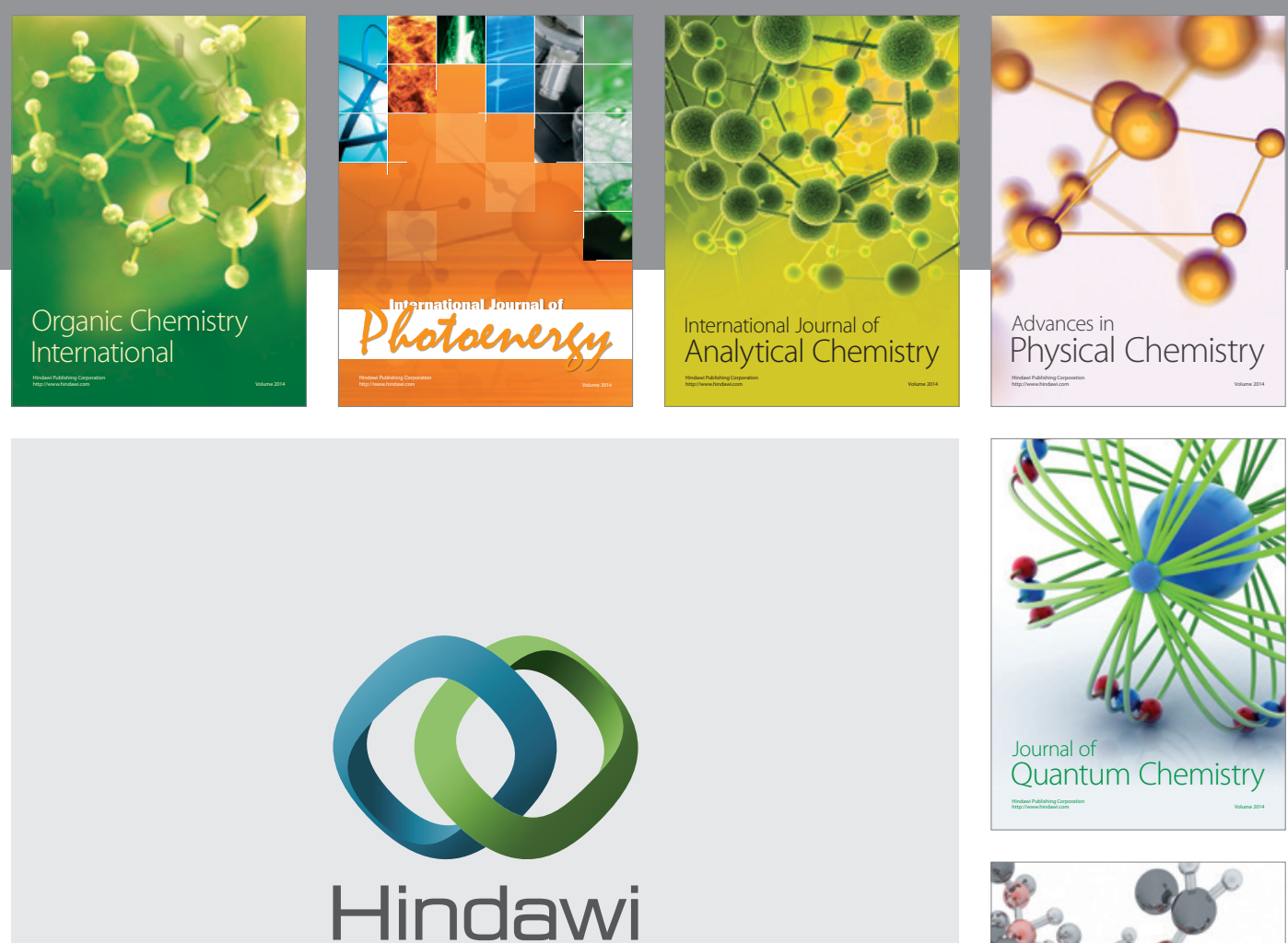

Submit your manuscripts at

http://www.hindawi.com

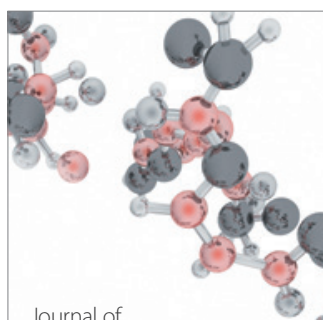

Analytical Methods

in Chemistry

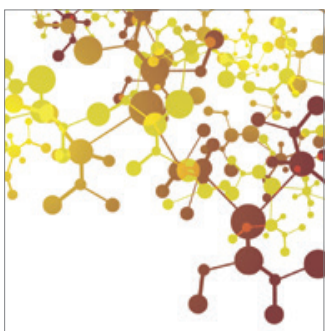

Journal of

Applied Chemistry

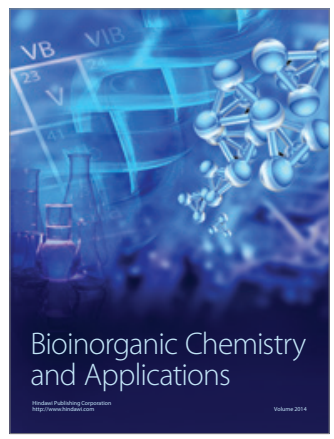

Inorganic Chemistry
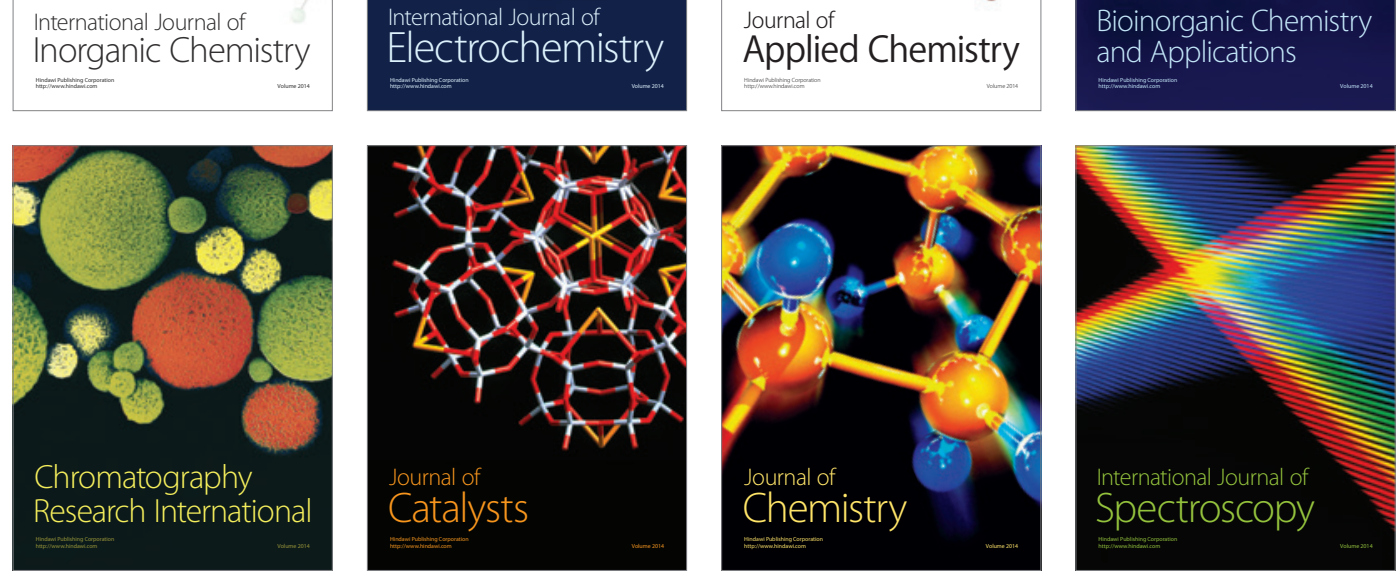